\title{
Knockdown of the T-box transcription factor Brachyury increases sensitivity of adenoid cystic carcinoma cells to chemotherapy and radiation in vitro: Implications for a new therapeutic principle
}

\author{
YOSUKE KOBAYASHI $^{1 *}$, TSUYOSHI SUGIURA ${ }^{2 *}$, IKUMI IMAJYO ${ }^{1}$, MIYUKI SHIMODA ${ }^{1}$, \\ KOTARO ISHII $^{1}$, NAONARI AKIMOTO ${ }^{1}$, NAOYA YOSHIHAMA ${ }^{1}$ and YOSHIHIDE MORI ${ }^{1}$
}

\begin{abstract}
Sections of ${ }^{1}$ Oral and Maxillofacial Surgery, ${ }^{2}$ Oral and Maxillofacial Oncology, Division of Maxillofacial Diagnostic and Surgical Sciences, Faculty of Dental Science, Kyushu University, Higashi-ku, Fukuoka 812-8582, Japan
\end{abstract}

Received December 2, 2013; Accepted January 24, 2014

DOI: 10.3892/ijo.2014.2292

\begin{abstract}
Adenoid cystic carcinoma (AdCC) is highly metastatic and resistant to chemotherapy and radiotherapy. Recently, we reported that the T-box transcription factor Brachyury is a potential regulator of cancer stem cells (CSCs). Specifically, growth of CSCs was found to be controlled by Brachyury knockdown in AdCC. Since CSCs are resistant to chemotherapy and radiotherapy, this finding provides a new principle for therapies targeting CSCs. In the present study, we established that Brachyury knockdown suppresses chemoresistance and radioresistance in vitro. Brachyury was knocked down by transfecting Brachyury short hairpin RNA (shRNA) into the AdCC CSC cell line ACCS-M GFP. Brachyury knockdown significantly inhibited cell migration and invasion and suppressed chemoresistance. A quantitative PCR array of drug transporter genes revealed that knockdown of Brachyury caused downregulation of ATP-binding cassette transporter genes. Furthermore, ACCS-M GFP radioresistance was significantly suppressed by Brachyury knockdown. Knockdown of Brachyury significantly sensitized ACCS-M GFP cells to chemoradiotherapy. This study demonstrates that Brachyury knockdown reduces invasiveness and chemoresistance and radioresistance of CSCs in vivo. Therefore, Brachyury knockdown may be a useful therapeutic tool for sensitizing CSCs to conventional chemoradiotherapy.
\end{abstract}

\section{Introduction}

Adenoid cystic carcinoma (AdCC) is among the most common malignant tumors of the salivary glands and is characterized

Correspondence to: Dr Tsuyoshi Sugiura, Section of Oral and Maxillofacial Oncology, Division of Maxillofacial Diagnostic and Surgical Sciences, Faculty of Dental Science, Kyushu University, 3-1-1 Maidashi, Higashi-ku, Fukuoka 812-8582, Japan

E-mail: sugiura@dent.kyushu-u.ac.jp

${ }^{*}$ Contributed equally

Key words: adenoid cystic carcinoma cells, Brachyury, chemotherapy, radiation, cancer stem cell by unique clinical features and behavior. Although slow growing, AdCC spreads relentlessly into adjacent tissues. It carries a high risk of recurrence and distant metastases, with $40-60 \%$ of afflicted patients developing distant metastases to the lungs, bone, and soft tissues $(1,2)$. AdCC is resistant to chemotherapy and radiotherapy. Therefore, treatment-resistant distant metastases remain a significant obstacle to the longterm cure of patients with AdCC, emphasizing the need for anti-metastasis therapy for AdCC.

We previously established 3 AdCC cell lines that express green fluorescent protein (GFP) from the ACCS cell line using orthotopic transplantation and in vivo selection in the nude mouse. These 3 lines include the parental ACCS GFP, the highly tumorigenic ACCS-T GFP, and the metastatic ACCS-M GFP line. We demonstrated that ACCS-M GFP cells exhibited a loss of E-cadherin and integrins and a gain in vimentin, suggesting that the epithelial-mesenchymal transition (EMT) is a key event in AdCC metastasis that induces tumor cell dissemination from the primary tumor site (3). We also showed a direct correlation between EMT and prevalence of cancer stem cell-like cells in AdCC (4).

The EMT program triggered during tumor progression appears to be controlled by expression of early embryonic genes, including Twist, Snail, Slug, Goosecoid and SIP1 $(5,6)$. The transcription factors encoded by these genes impart mesenchymal traits to tumor cells, including motility and invasiveness. For example, expression of Twist is elevated in various cancers, including breast, prostate, gastric and melanoma (7). In addition, the T-box transcription factor Brachyury, a protein required for mesoderm formation during development (8-10), reportedly promotes EMT in human carcinoma cell lines (11). The latter study also showed that Brachyury overexpression in human carcinoma cells induced changes characteristic of EMT. These findings suggest that the EMT in cancer cells is controlled by mechanisms similar to the EMT during normal human development.

Other studies using neoplastic tissue have identified selfrenewing, stem-like cells within tumors, referred to as cancer stem cells (CSCs). CSCs constitute a minority of neoplastic cells within a tumor and are defined operationally by their ability to seed new tumors. For this reason, they have also 
been termed tumor-initiating cells (12). During the process of tumor metastasis, which is often enabled by EMT (13), disseminated cancer cells are thought to require self-renewal properties similar to those exhibited by stem cells in order to spawn macroscopic metastases. This raises the possibility that the EMT process, which enables cancer cell dissemination, may also impart self-renewal to disseminating cancer cells. Indeed, emerging evidence of a direct interaction between EMT and CSCs $(11,14,15)$. Similarly to normal stem cells, CSCs are regulated by key genes, such as Oct4, Nanog, $c-M y c$, Sox2, and Klf4, which are similar to EMT-regulator genes $(16,17)$. CSCs are resistant to chemotherapy and radiotherapy $(18,19)$, suggesting a new therapeutic principle for targeting CSCs $(20,21)$.

We have confirmed a direct interaction between the EMT and CSCs in the highly metastatic AdCC subclone ACCS-M GFP. We also reported that the T-box transcription factor Brachyury, which is also a marker of mesoderm differentiation $(22,23)$, regulates CSC and the EMT in AdCC cells. Brachyury knockdown exerted a stronger effect on cancer stemness and EMT phenotype than did knockdown of the conventional CSC regulator gene, Sox2. By reducing the stemness of CSCs, Brachyury knockdown significantly inhibited tumorigenicity and metastasis in vivo (4). This hypothesis has been supported by recent evidence linking Brachyury to CSCs in colon cancer (24).

These observations suggest that knocking down Brachyury can control CSC and EMT, thus inducing CSC differentiation and sensitization to conventional chemotherapy and radiotherapy. In this study, we validated that Brachyury knockdown suppresses chemo- and radioresistance in vitro as a first step in establishing its therapeutic potential against CSCs.

\section{Materials and methods}

Chemicals. Standard anticancer drug kits were provided by Scientific Support Programs for Cancer Research, Grantin-Aid for Scientific Research on Innovative Areas from the Ministry of Education, Culture, Sports, Science and Technology of Japan. Docetaxel, 5-fluorouracil (5-FU), paclitaxel, cisplatin (CDDP), mitomycin $\mathrm{C}$, bestatin hydrochloride, bleomycin sulfate and etoposide were purchased from Sigma-Aldrich (St. Louis, MO, USA). Actinomycin D and streptomycin-SP were purchased from Calbiochem (Merck, Darmstadt, Germany).

Cells and cell culture. The human cell lines ACCS, ACCS GFP and ACCS-M GFP were established in our laboratory as previously described (3). Briefly, the parental cell line ACCS and the GFP-transfected sub-line ACCS GFP displayed similar morphology, growth rate and tumorigenicity in vitro and in vivo. Similar to the parental ACCS cells, ACCS GFP cells had low tumorigenicity (22.2\% incidence). Using ACCS GFP cells injected into the tongue of nude mice, tumor formation was observed under the excitation wavelength. Green fluorescence was not observed in the absence of tumors. We performed in vivo selection of clones with higher tumorigenicity by repeatedly recovering cells in vitro and transplanting them into the tongue of nude mice. This selection process yielded a subline exhibiting high tumorigenicity (100\% incidence) and high frequency of metastasis to submandibular lymph nodes $(100 \%$ incidence); these cells were termed ACCS-M GFP. The histological and immunohistochemical features of ACCS-M GFP tumors were similar to the solid pattern of AdCC. The cell lines were maintained as a monolayer culture in Dulbecco's modified Eagle's medium (DMEM; Sigma-Aldrich) supplemented with $10 \%$ fetal bovine serum (FBS; ICN Biomedicals, Aurora, OH, USA), 2 mM l-glutamine, penicillin G, and streptomycin in a humidified incubator under an atmosphere of 5\% $\mathrm{CO}_{2}$ at $37^{\circ} \mathrm{C}(3)$.

Transfection of Brachyury and SOX2 shRNA. Cultured ACCS cells were transfected with short hairpin RNA (shRNA) lentiviral plasmids (pLKO.1-puro; Sigma-Aldrich) using Lipofectamine LTX (Invitrogen Life Technologies, Carlsbad, CA, USA) according to the manufacturer's instructions as previously described (4). ACCS-M sh cont. cells were generated by transfecting ACCS-M GFP cells with pLKO.1-puro Control shRNA Vector (Sigma-Aldrich). ACCS-M shBr and ACCS-M shSOX2 cells were generated by transfecting ACCS GFP and ACCS-M GFP cells with pLKO.1-puro/sh. Brachyury or pLKO.1-puro/sh. SOX2 (Sigma-Aldrich), respectively. Colonies resistant to puromycin (Sigma) were pooled from the individual transfection experiments. The expression level of Brachyury in shRNA-transfected ACCS cells was monitored by real-time reverse transcription-PCR (RT-PCR) (4). All transfected cells were maintained in DMEM containing $10 \%$ FBS and $2 \mu \mathrm{g} / \mathrm{ml}$ puromycin (Sigma-Aldrich).

Wound healing assay. Cells $\left(3 \times 10^{5}\right)$ were seeded on a 6-well plate. After $24 \mathrm{~h}$, 'wounds' were scratched with a 200- $\mu 1$ pipette tip, washed with medium and observed under a fluorescence microscope (BZ-8000; Keyence, Osaka, Japan). The wound regions were photographed again after 8,16 and $24 \mathrm{~h}$, and the wound areas were measured. Wound area was calculated using the following formula: wound area (\% of control $)=($ wound area after the indicated period $\mathrm{x} 100) /$ initial wound area.

Evaluation of tumor dissemination from the primary cancer nest. Evaluation of tumor dissemination from the primary cancer nest was performed as previously described (25). Briefly, living ACCS cell lines were fluorescently labeled using Vybrant DiO and DiD cell-labeling solutions (Molecular Probes, Eugene, OR, USA) according to the manufacturer's instructions. Then, $1 \times 10^{6}$ labeled cells were pelleted and resuspended in $10 \mu \mathrm{l}$ collagen type I gel to form a solid cell cluster. The collagen-embedded tumor cell pellets were allowed to solidify for $30 \mathrm{~min}$ at $37^{\circ} \mathrm{C}$ in a $100-\mu 1$ microcentrifuge tube; the pellets were then embedded in non-labeled fibroblasts containing collagen type I gel $\left(1 \times 10^{5}\right.$ cells $\left./ \mathrm{ml}\right)$ and solidified. Growth medium was placed over the collagen gels and cultured. Tumor dissemination was observed under a fluorescence microscope (BZ-8000; Keyence). The grade of tumor dissemination from the tumor cell pellet (modeling the primary tumor nest) was evaluated by measuring the distance of all cells from the edge of the nest in 5 randomly selected, standardized rectangular light fields (500x100 $\mu \mathrm{m})$, and the values were summed. The evaluation was conducted twice daily for 7 days. 
A

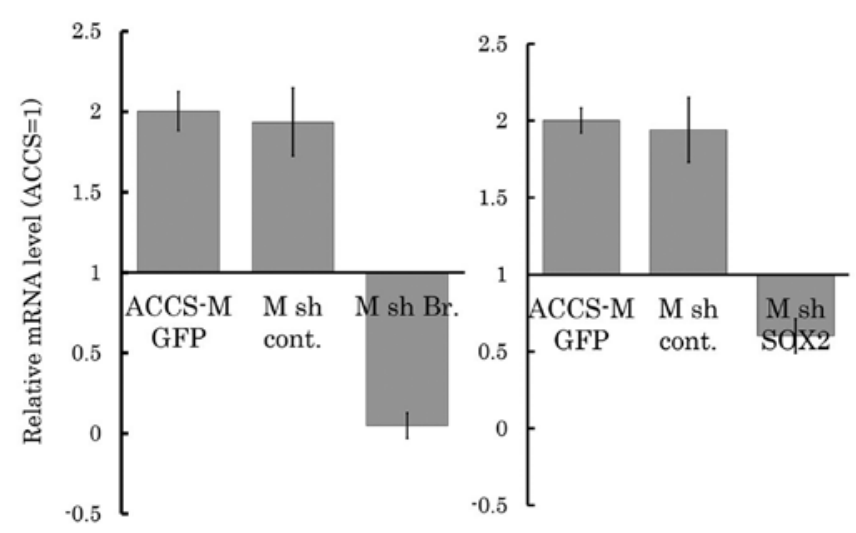

B

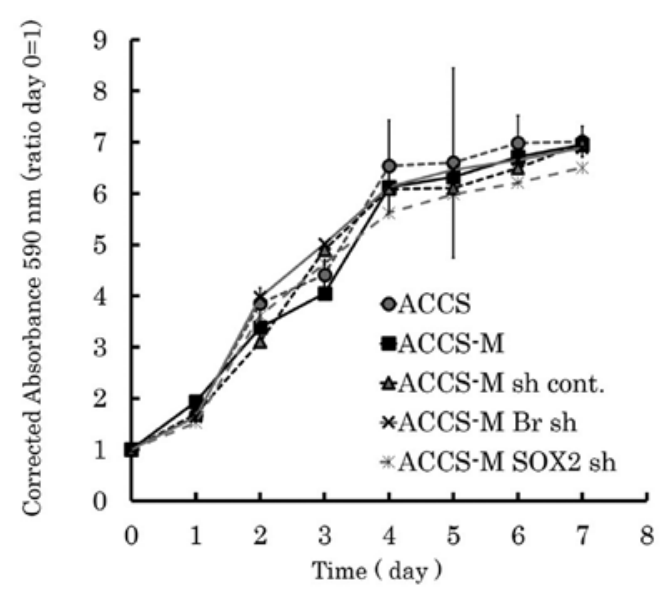

Figure 1. Effect of Brachyury and SOX2 knockdown on ACCS cell growth. (A) The mRNA expression levels of Brachyury and Sox2 in shRNA-transfected cells as quantified by real-time RT-PCR. Data are shown as mRNA levels relative to ACCS GFP. Error bars indicate the standard deviation. (B) Cell growth of ACCS and its derivatives, as evaluated by MTT assay. Error bars indicate the standard deviation.

MTT assay. ACCS cell lines were seeded into CellTiter 96 Aqueous Non-radioactive Cell Proliferation Assay G4000 plates (Promega, Madison, WI, USA) at a density of $5 \times 10^{3}$ cells per well and incubated in DMEM containing 10\% FBS for $8 \mathrm{~h}$. The medium was replaced with serum-free DMEM after 3 washes with PBS. For chemosensitivity analysis, a dilutional series of anticancer drugs was applied at final drug concentrations of $0,0.001,0.01,0.1,1,10,100$ and $1,000 \mu \mathrm{M}$ and incubated for $24 \mathrm{~h}$ in a humidified incubator under an atmosphere of $5 \% \mathrm{CO}_{2}$ at $37^{\circ} \mathrm{C}$. For radiosensitivity analysis, in vitro gamma-ray irradiation was administered at $5,10,15$, or $30 \mathrm{~Gy}$ with a Gammacell $40^{\circledR}$ Exactor Low Dose-Rate Research Irradiator (Best Theratronics, Ottawa, Canada), and cells were then incubated for 48 or $72 \mathrm{~h}$ in a humidified incubator under an atmosphere of $5 \% \mathrm{CO}_{2}$ at $37^{\circ} \mathrm{C}$. After incubation, ACCS cells were analyzed by CellTiter 96 Aqueous Non-radioactive Cell Proliferation Assay G4000 (Promega) according to the manufacturer's instructions. The absorbance of samples at $590 \mathrm{~nm}$ (A590) was measured with a microplate reader (Model 680, Bio-Rad, USA). All experiments were carried out in triplicate and repeated 3 times.
Data were normalized to the untreated controls and reported as $\%$ viability. The $\mathrm{IC}_{50}(\mu \mathrm{M})$ values for cytotoxicity of the anticancer drug represents the concentration yielding $50 \%$ viability, which was determined from the concentrationviability curve. The concentration-viability curve was generated using a non-linear regression model with the Solver function of Microsoft Excel as previously described (26).

Real-time RT-PCR. Total RNA was extracted from ACCS GFP cells using the RNeasy Mini kit (Qiagen, Chatsworth, CA, USA) and used for first-strand cDNA synthesis. The mRNA levels were quantified in triplicate using a real-time PCR system with the Brilliant SYBR Green qPCR kit (Stratagene, La Jolla, CA, USA) for Brachyury and Sox2 or the $\mathrm{RT}^{2}$ Profiler PCR Array (96-well format) for human drug transporters (Qiagen). Specific primers for Brachyury and Sox2 were: hBrachyury (F) 5'-TGCTGCAATCCCATGACA-3', (R) 5'-CGTTGCTCACAGACCACA-3'; hSOX2, (F) 5'-TGG GTTCGGTGGTCAAGT-3', (R) 5'-CTCTGGTAGTGCTG GGACA-3'. The PCR cycling conditions were $10 \mathrm{~min}$ at $95^{\circ} \mathrm{C}$ followed by $47 \mathrm{cycles}$ at $95^{\circ} \mathrm{C}$ for $30 \mathrm{sec}, 60^{\circ} \mathrm{C}$ for $30 \mathrm{sec}$, and $72^{\circ} \mathrm{C}$ for $60 \mathrm{sec}$. Dissociation curve analyses confirmed that the signals corresponded to unique amplicons. Expression levels were normalized to $\beta$-actin mRNA levels for each sample obtained from parallel assays and analyzed using the LightCycler software package version 3.5 (Roche Diagnostics, Mannheim, Germany) for hBrachyury and hSOX2 and Mx 3000P QPCR system (Agilent Technologies, CO, USA) for the $\mathrm{RT}^{2}$ Profiler PCR Array.

Statistical analysis. All data are represented as mean $\pm \mathrm{SD}$, as analyzed via analysis of variance and Student's t-test, and processed using the statistical software SPSS 13.0. Statistical significance was defined as $\mathrm{P}<0.05$.

\section{Results}

Brachyury and SOX2 shRNA do not influence growth of ACCS cell lines. We established ACCS GFP and ACCS-M GFP-derived cell lines by stable transfection of Brachyury or SOX2 shRNA lentiviral plasmids. The expression level of Brachyury or SOX2 in shRNA-transfected ACCS cells was monitored by real-time RT-PCR to confirm silencing of the target genes (Fig. 1A). We first analyzed the effect of Brachyury or SOX2 knockdown on cell growth in vitro by MTT assay. Cancer stem cell-like ACCS-M GFP cells demonstrated a similar growth pattern to parental ACCS GFP cells. Stable transfection of shRNA did not affect cell growth (ACCS-M sh cont. GFP). Neither Brachyury shRNA nor SOX2 shRNA affected cell growth (ACCS-M shBr GFP and ACCS-M shSOX2 GFP, respectively; Fig. 1B).

Brachyury shRNA inhibits cell migration. The effect of Brachyury or SOX2 knockdown on cell migration in vitro was analyzed by the wound healing assay (Fig. 2). Cell migration of ACCS-M GFP cells was approximately twice as fast as that of ACCS GFP cells. Brachyury knockdown significantly inhibited migration of ACCS-M GFP cells to the level of parental ACCS GFP $(\mathrm{P}=0.001)$. By contrast, SOX2 knockdown had no effect on ACCS-M GFP cell migration. 

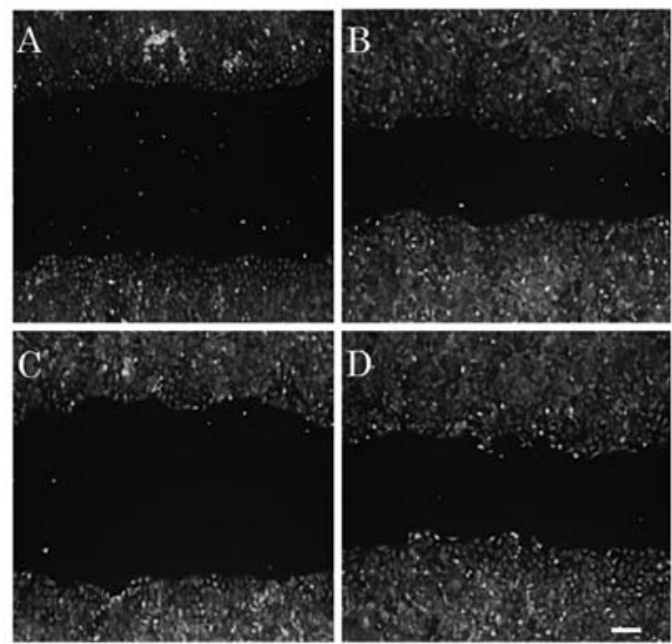

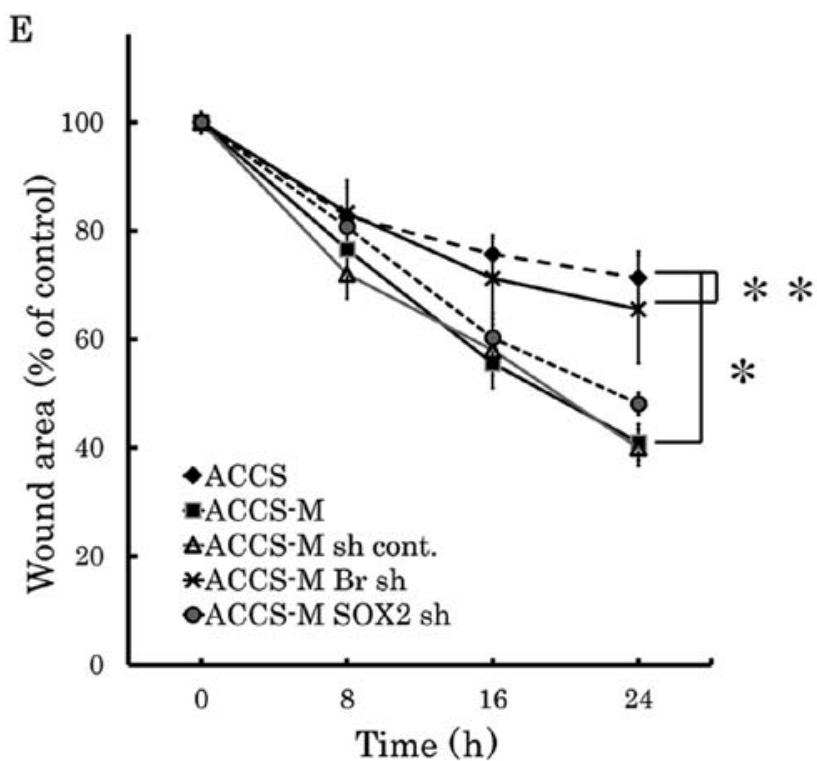

Figure 2. Effect of Brachyury and SOX2 knockdown on ACCS cell migration. Cell migration, as evaluated by the wound-healing assay. Photomicrographs of wound regions $24 \mathrm{~h}$ after the start of the assay (A-D). ACCS GFP (A), ACCS-M GFP (B), ACCS-M shBr GFP (C), and ACCS-M shSOX2 GFP (D) cells. Bar, $100 \mu \mathrm{m}$. (E) Calculated wound area. ${ }^{*} \mathrm{P}<0.001,{ }^{* *} \mathrm{P}<0.05$.
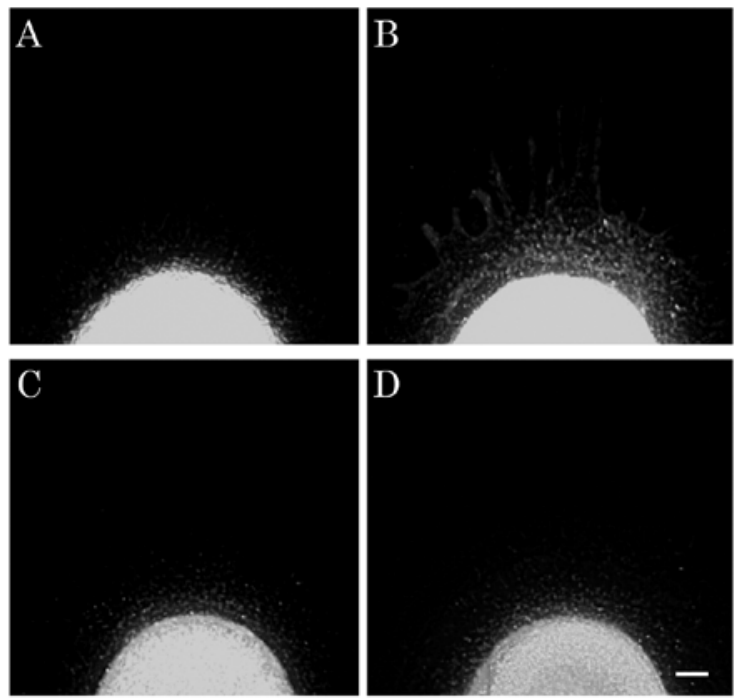

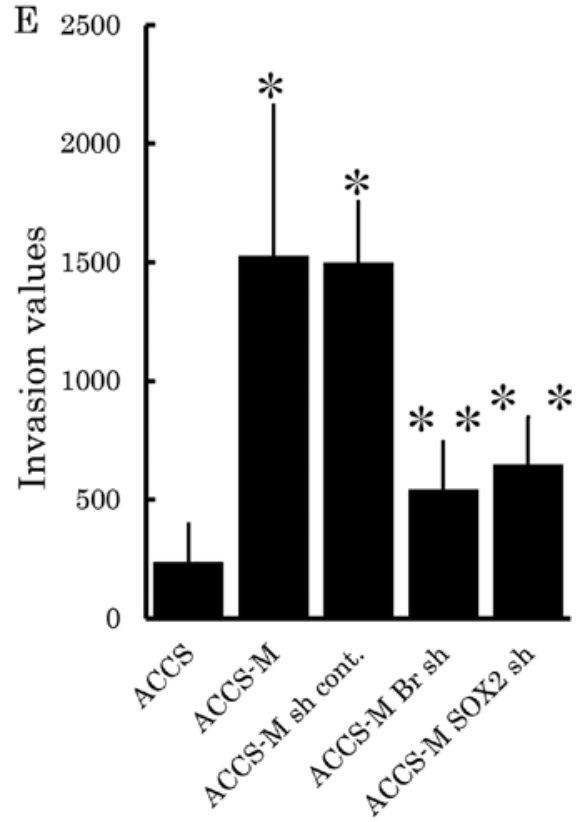

Figure 3. Effect of Brachyury and SOX2 knockdown on ACCS cell invasiveness. ACCS GFP (A), ACCS-M GFP (B), ACCS-M shBr GFP (C), and ACCS-M shSOX2 GFP (D) cells as analyzed with the new invasion assay. Photomicrographs taken after a 7-day incubation. Bar, $100 \mu \mathrm{m}$. (E) Error bars indicate standard deviation. ${ }^{*} \mathrm{P}<0.001,{ }^{* *} \mathrm{P}<0.05$.

Brachyury and SOX2 shRNA inhibit cell invasion. We next analyzed the effect of Brachyury or SOX2 knockdown on cell invasiveness in vitro using our previously reported tumor-cell dissemination assay (25). In this assay, invasion of carcinoma cells is visualized as small green fluorescent spots escaping from a cell pellet that models the primary cancer nest. Therefore, we evaluated cancer cell invasion by the number of invasive cells and their distance from the artificial primary cancer nest. As shown in Fig. 3B, ACCS-M GFP cells demonstrated aggressive cell invasion into artificial stromal tissue. Invasiveness of ACCS-M GFP cells was strongly inhibited by knockdown of Brachyury (Fig. 3C) or SOX2 (Fig. 3D). Fig. 3E compares invasiveness among ACCS cell lines. Relative invasiveness values (ACCS GFP = 1) were 6.4 (ACCS-M GFP), 2.3 (ACCS-M shBr GFP), and 3.2 (ACCS-M shSOX2 GFP).

Brachyury and SOX2 shRNA induce chemosensitivity in vitro. Cancer stem cells are known to resist various types of anticancer drugs. Therefore, we next assessed whether knockdown of cancer stem cell regulators could change their sensitivity to anticancer drugs. ACCS-M GFP cells demonstrated chemoresistance to CDDP, docetaxel, actinomycin D, etoposide, 5-FU, 

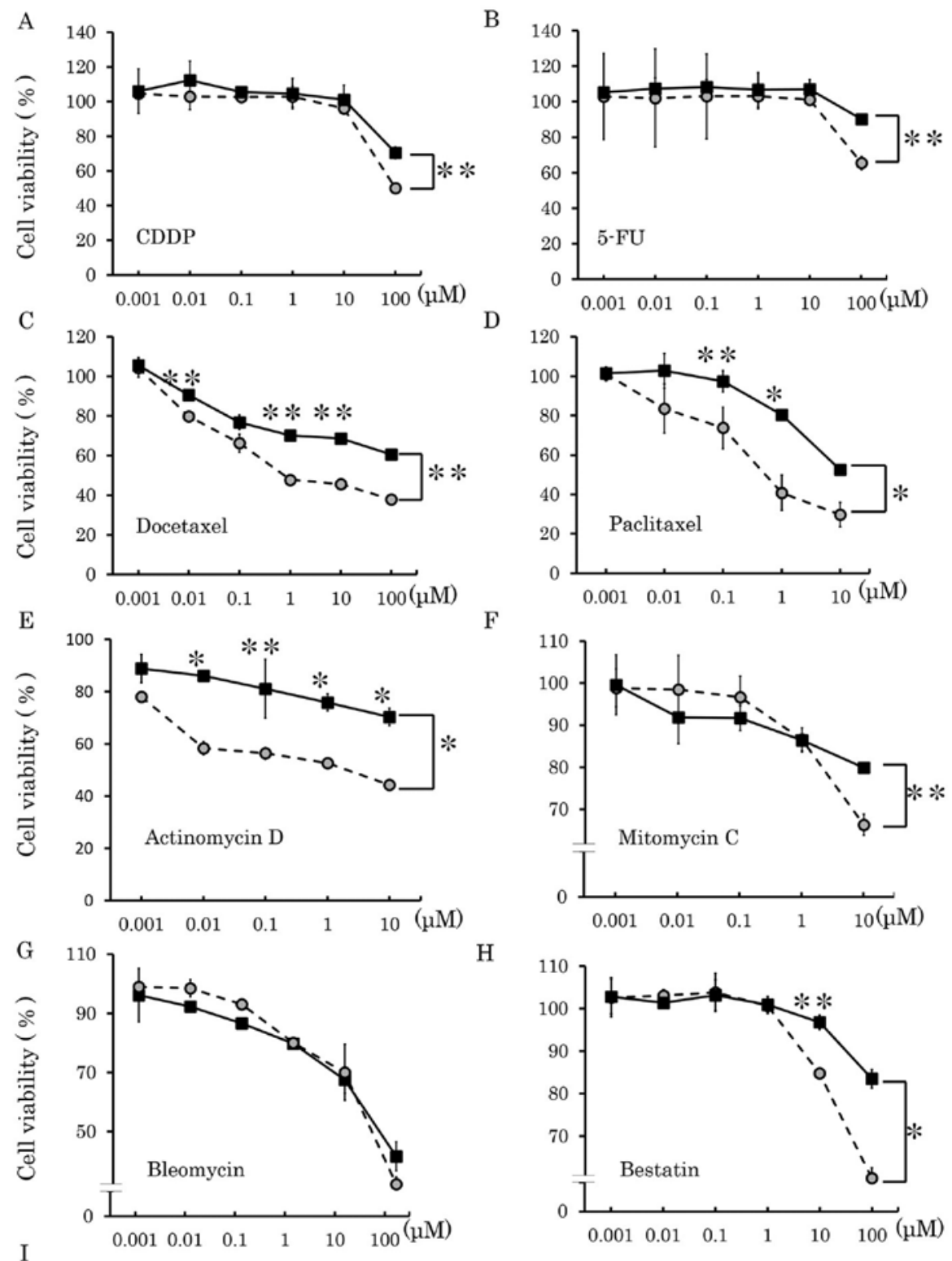

$\mathrm{H}$
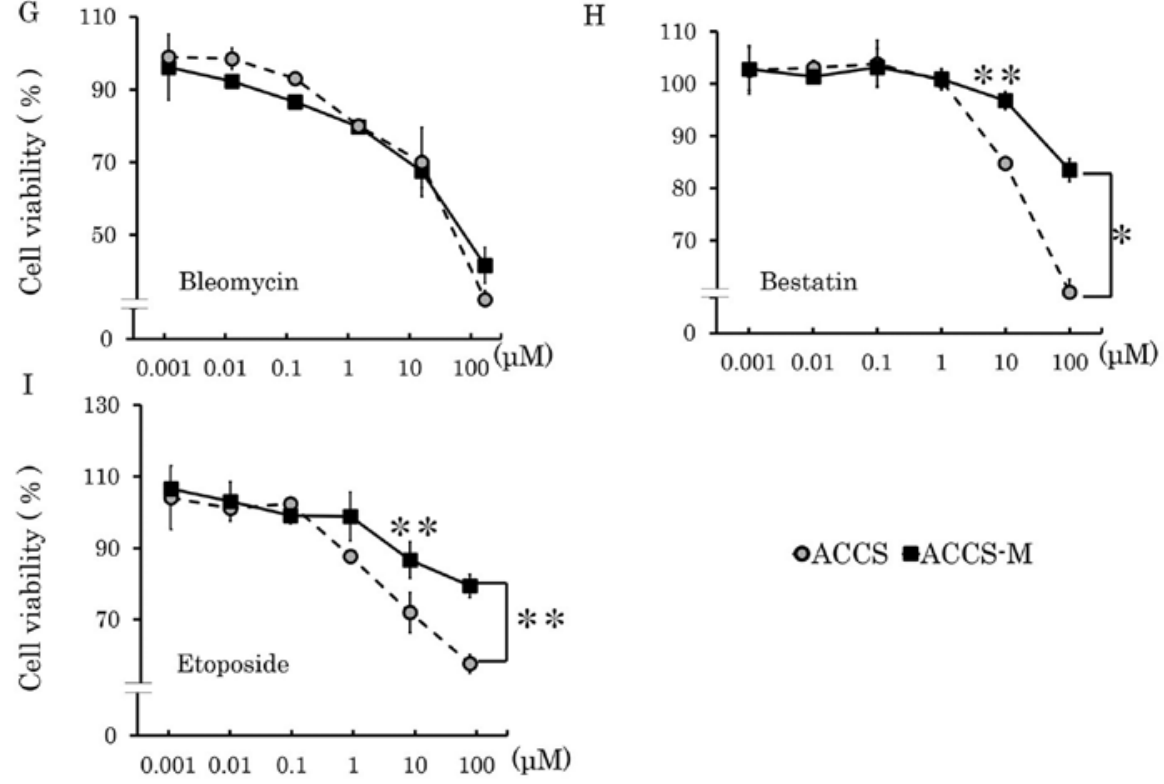

OACCS $=\mathrm{ACCS}-\mathrm{M}$

Figure 4. Comparison of chemosensitivity between ACCS GFP and ACCS-M GFP cells (A-1). Cell viability after application of anticancer drugs to ACCS GFP and ACCS-M GFP cells at final concentrations of $0,0.001,0.01,0.1,1,10$, or $100 \mu \mathrm{M}$. Error bars indicate standard deviation. "P<0.001, ${ }^{* *} \mathrm{P}<0.05$.

paclitaxel, mitomycin $\mathrm{C}$ and bestatin (Fig. 4). Table I shows the $\mathrm{IC}_{50}$ values of each anticancer drug in each ACCS cell line. The $\mathrm{IC}_{50}$ values of ACCS-M GFP cells were higher than those of ACCS GFP cells for each anticancer agent (range: 1.2-355fold). In particular, resistance to taxane drugs, docetaxel and paclitaxel, was very high (355- and 23-fold of ACCS GFP IC 50 values, respectively). Brachyury shRNA and SOX2 shRNA reduced chemoresistance of ACCS-M GFP cells, but the effect of Brachyury shRNA was greater than that of SOX2
shRNA (Fig. 5 and Table I). Relative $\mathrm{IC}_{50}$ values (ACCS-M GFP $=1$ ) of Brachyury shRNA were $\sim 0.33$ (docetaxel) to 0.85 (mitomycin C and bleomycin), and those of SOX2 shRNA were 0.59 (bestatin) to 1 (paclitaxel, mytomycin $\mathrm{C}$, and bleomycin). However, with the exception of bestatin, the degree of reduction did not reach parental ACCS GFP levels (Table I). We could not determine an $\mathrm{IC}_{50}$ for etoposide, because ACCS cell lines showed strong resistance, and the maximum dose of etoposide $(1,000 \mu \mathrm{M})$ did not reduce cell viability to $50 \%$. 


$$
\text { A }
$$
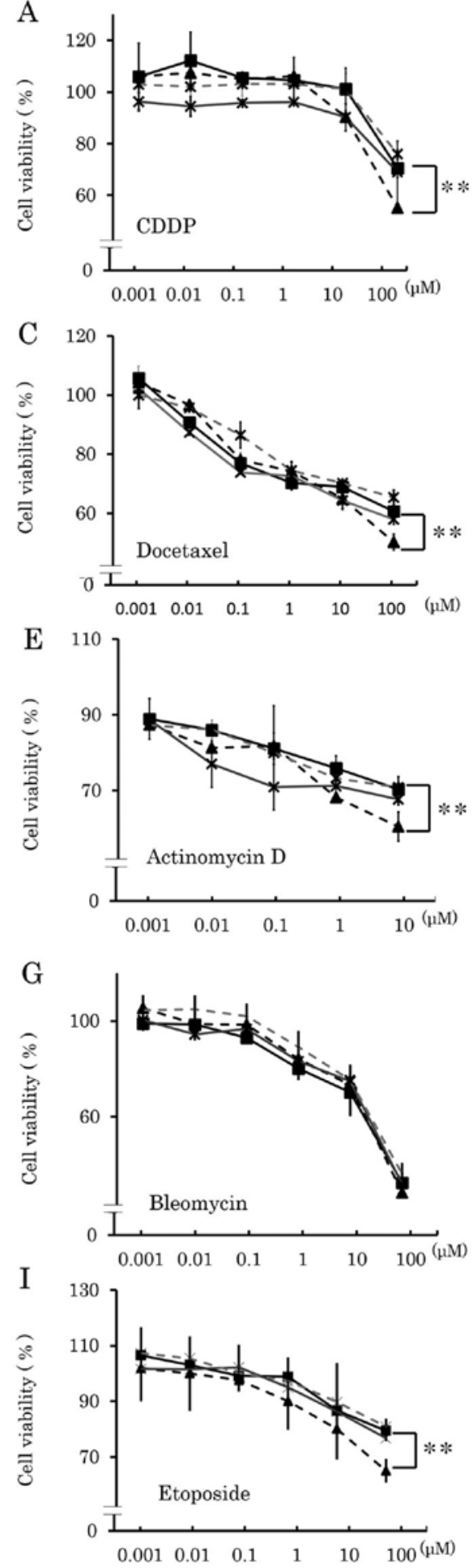

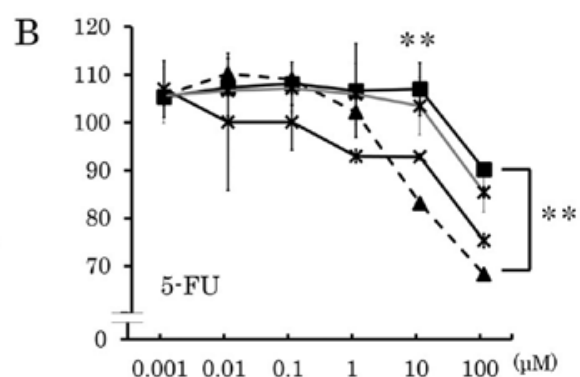

D

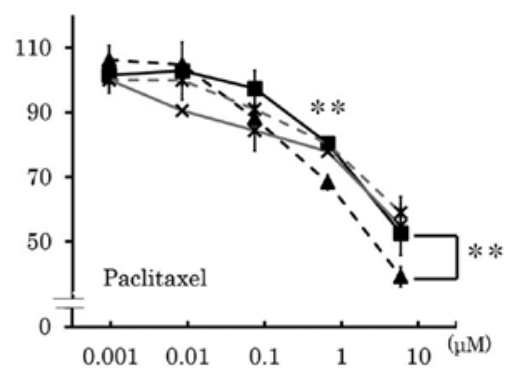

F

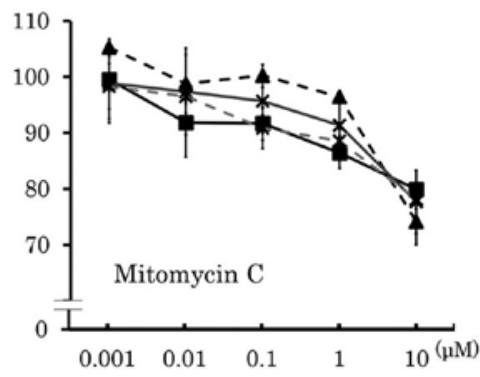

$\mathrm{H}$

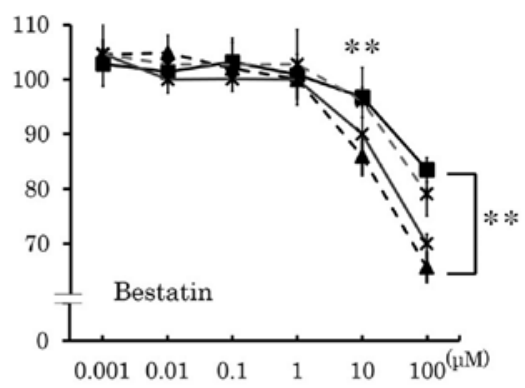

-ACCS-M

$\triangle \mathrm{ACCS}-\mathrm{M} \mathrm{Br}$ sh

*ACCS-M SOX2 sh

*ACCS-M sh cont.

Figure 5. Effect of Brachyury and SOX2 knockdown on chemosensitivity of ACCS-M GFP cells (A-I). Cell viability after application of various types and concentrations of anticancer drugs to ACCS-M GFP, ACCS-M sh cont., GFP ACCS-M shBr GFP, and ACCS-M shSOX2 GFP cells. Error bars indicate standard deviation. ${ }^{* *} \mathrm{P}<0.05$.

Brachyury and SOX2 shRNA modify expression of drugtransporter genes in vitro. Multidrug resistance in cancer is heavily dependent on 2 major superfamilies of membrane transporter proteins that influence the pharmacokinetics of drugs, ATP-binding cassette (ABC) transporters and solutecarrier (SLC) transporters. Therefore, we analyzed the effect of Brachyury and SOX2 knockdown on the expression levels of these membrane transporters by real-time PCR array.
The differences between ACCS-M GFP and ACCS GFP in expression of these membrane transporters were not significant (1.05-1.2-fold). Notably, ACCS-M GFP cells generally expressed higher levels of $\mathrm{ABC}$ transporter genes than ACCS GFP cells, and this expression was reduced by Brachyury or SOX2 knockdown (Fig. 6A). SLC transporter genes were also generally expressed at higher levels in ACCS-M GFP cells with the exception of SLC19A1 and SLC5A1. Brachyury 
A

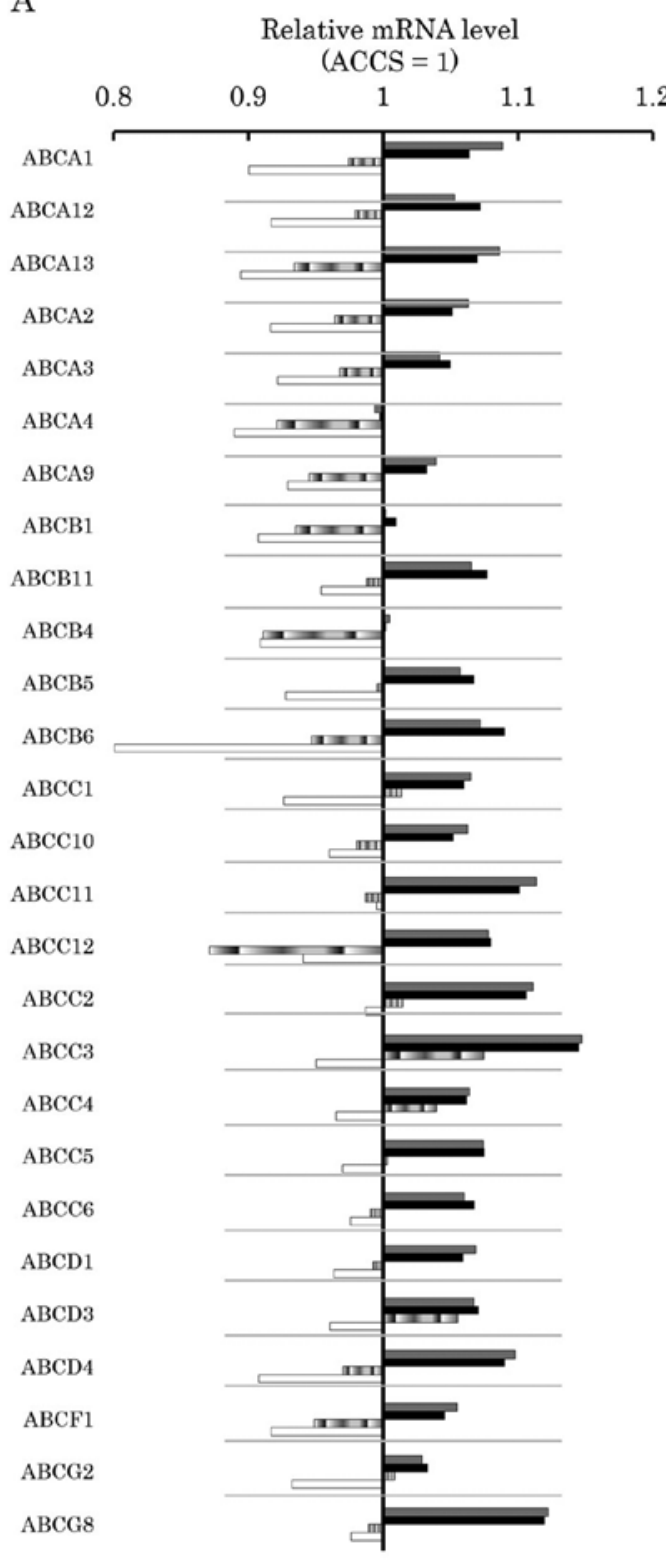

$\mathrm{B}$

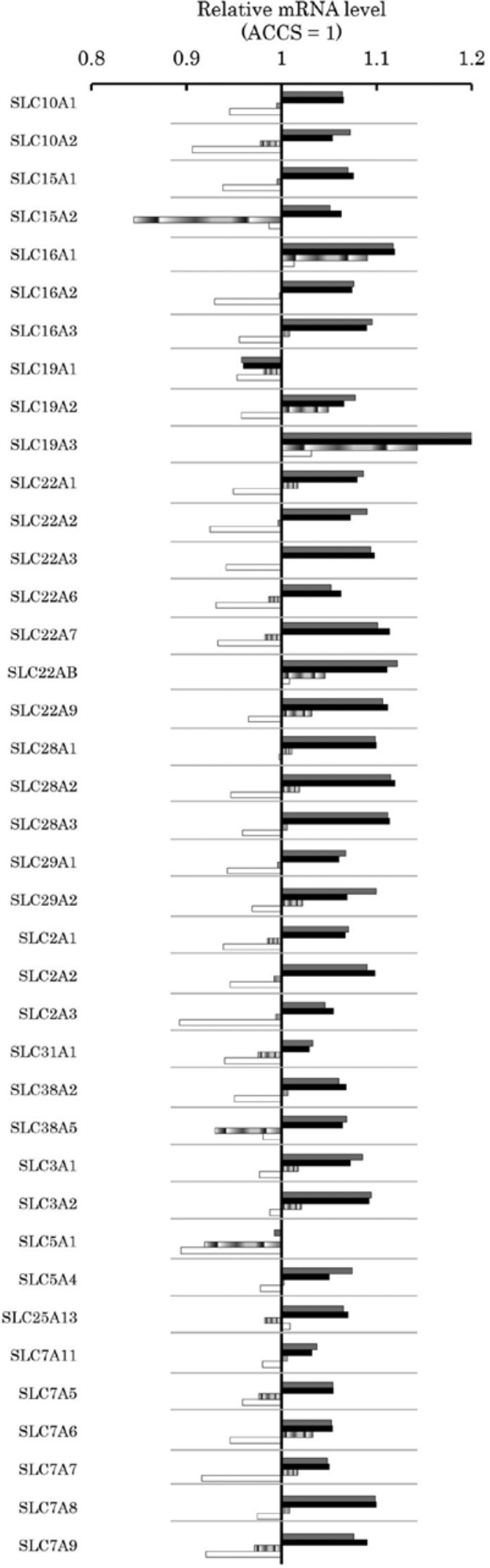

Figure 6. Effect of Brachyury and SOX2 knockdown on mRNA expression of drug transporters. mRNA expression levels of the indicated genes in ACCS-M GFP cells and derivatives as quantitated by real-time RT-PCR. mRNA levels are reported as mRNA relative tp ACCS GFP. The expression levels of ABC transporter genes (A) and SLC family genes (B) are shown. Error bars indicate standard deviation.

knockdown increased the expression of only one SLC transporter gene, SLC19A1 (Fig. 6B).
Brachyury and SOX2 shRNA induce radiosensitivity in vitro. Cancer stem cells are insensitive to radiation. We analyzed 
Table I. Measured $\mathrm{IC}_{50}(\mu \mathrm{M})$ of each anticancer drug.

\begin{tabular}{lcccrr}
\hline & \multicolumn{5}{c}{$\mathrm{IC}_{50}(\mu \mathrm{M})$} \\
\cline { 2 - 6 } Anticancer drug & ACCS & ACCS-M & ACCS-M sh cont. & Br sh & SOX2 sh \\
\hline Cisplatin & 353.4 & 527.6 & 529.1 & 360.2 & 403.4 \\
5-Fluorouracil & 463.5 & 842.9 & 835.1 & 623.7 & 673.6 \\
Docetaxel & 0.9 & 320.5 & 315.8 & 104.5 & 248.5 \\
Paclitaxel & 0.75 & 17.3 & 18.5 & 6.7 & 18.8 \\
Actinomycin D & 3.9 & 43.6 & 42.1 & 27.5 & 35.5 \\
Mitomycin C & 33.8 & 49.8 & 47.1 & 40.2 & 48.2 \\
Bleomycin & 41.1 & 57.8 & 58.6 & 50.2 & 55.8 \\
Bestatin & 459 & 558.8 & 549.3 & 306.7 & 325 \\
Etoposide & N.D. & N.D. & N.D. & N.D. & N.D. \\
\hline
\end{tabular}

N.D., not determined.
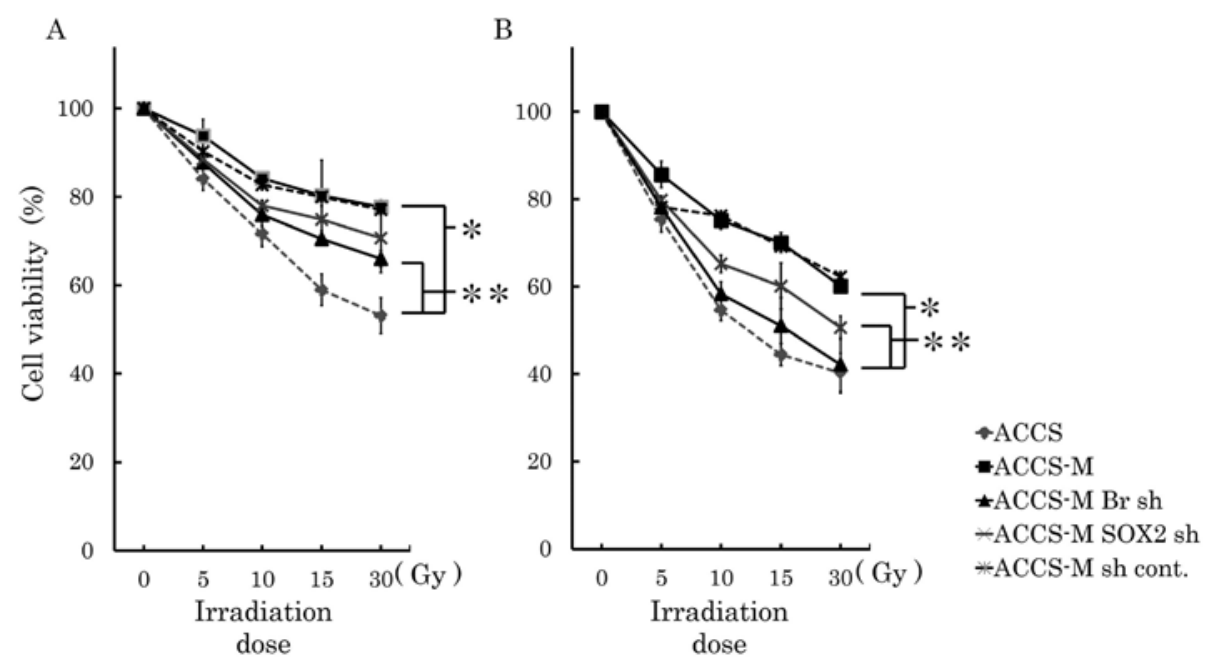

Figure 7. Effect of Brachyury and SOX2 knockdown on radiosensitivity of ACCS-M GFP cells. Cell viability after various doses of radiation in ACCS GFP, ACCS-M GFP, ACCS-M sh cont. GFP, ACCS-M shBr GFP, and ACCS-M shSOX2 GFP cells after $48 \mathrm{~h}$ (A) and $72 \mathrm{~h}$ (B). Error bars indicate the standard deviation. ${ }^{* *} \mathrm{P}<0.05$.

sensitivity to radiation treatment in vitro and found that ACCS-M GFP cells were significantly more resistant to irradiation than ACCS GFP cells $(\mathrm{P}<0.001)$. The viability of ACCS GFP and ACCS-M GFP cells was 53 and 77\%, respectively, $48 \mathrm{~h}$ after 30-Gy irradiation and 40 and $60 \% 72 \mathrm{~h}$ after 30-Gy irradiation, respectively. Brachyury and SOX2 knockdown reduced cell viability $72 \mathrm{~h}$ after $30-$ Gy irradiation. Brachyury knockdown reduced cell viability upon irradiation significantly more than SOX2 knockdown $(\mathrm{P}<0.05)$ and increased the radiosensitivity of ACCS-M GFP to the level of ACCS GFP cells (Fig. 7).

Brachyury and SOX2 shRNA enhance the cytotoxicity of concurrent chemoradiation treatment in vitro. We analyzed the effect of Brachyury and SOX2 shRNA on concurrent chemoradiation treatment (CRT) in vitro to assess the clinical potential of Brachyury and SOX2 shRNA. Fig. 8 compares anticancer drugs with or without radiation treatment. Brachyury and SOX2 shRNA demonstrated significantly affected CRT. Brachyury shRNA was more effective than SOX2 shRNA and significantly affected 5-FU, docetaxel, paclitaxel, mitomycin $\mathrm{C}$, and etoposide CRT $(\mathrm{P}<0.001)$. Notably, Brachyury shRNA was also significantly effective for CRT with bleomycin and mitomycin C (both $\mathrm{P}<0.05$ ), while Brachyury shRNA did not affect any single chemotherapy treatment.

\section{Discussion}

Clinically, oral AdCC is resistant to chemotherapy and radiotherapy, which poses a major obstacle to treatment. CSCs may contribute to chemo- and radioresistance, and new cancer therapies targeting CSCs are under investigation (27-29). Recently, we reported that T-box transcription factor Brachyury is a putative factor underlying the EMT and CSC stemness of AdCC in vitro and metastasis in vivo and that Brachyury knockdown inhibits AdCC tumorigenicity and metastasis (4). Therefore, clinical application of Brachyury knockdown may 

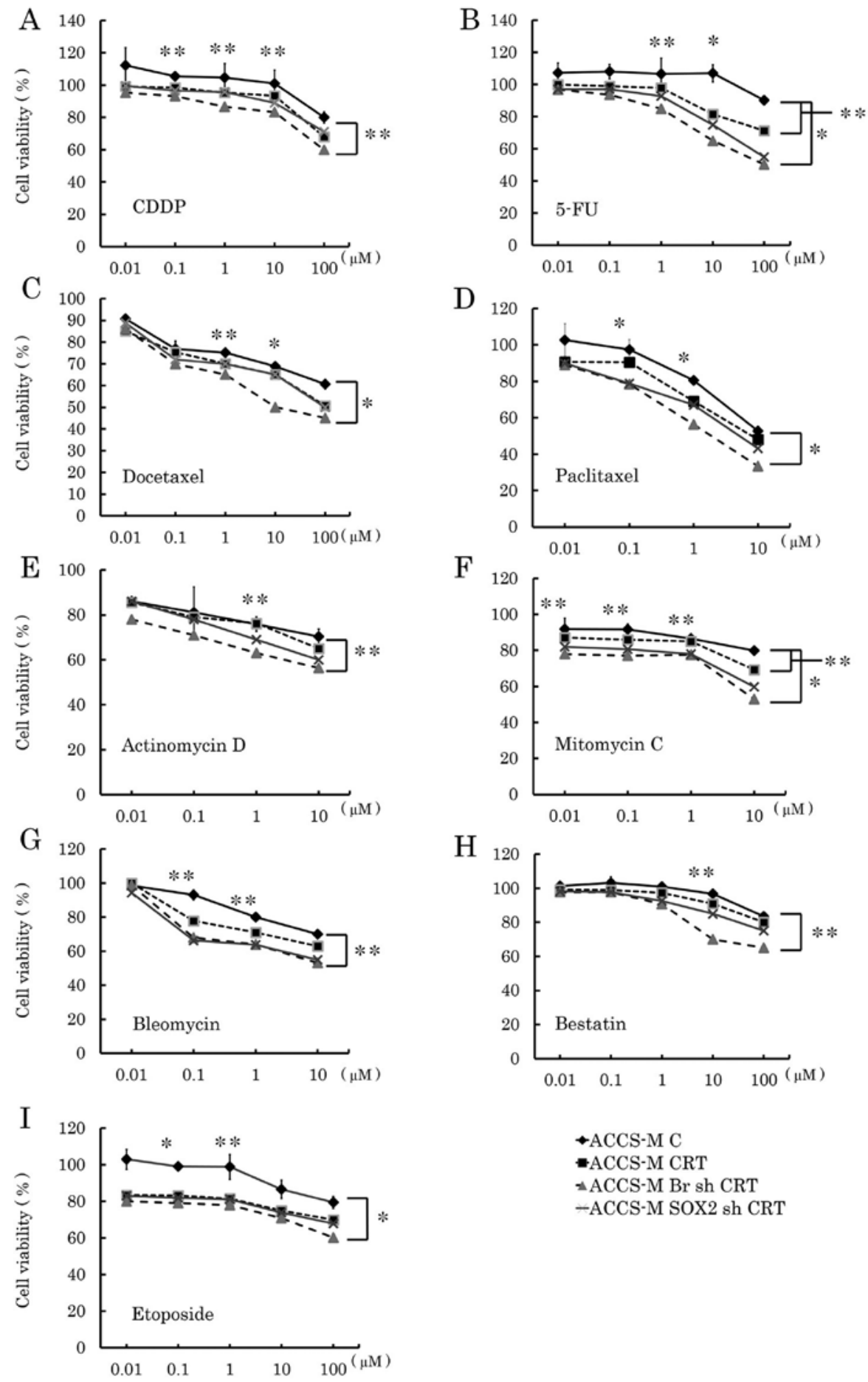

$\rightarrow \mathrm{ACCS}-\mathrm{M} \mathrm{C}$

-ACCS-M CRT

$\leadsto$ ACCS-M Br sh CRT

-ACCS-M SOX2 sh CRT

Figure 8. Effect of Brachyury and SOX2 knockdown on chemoradiation treatment of ACCS-M GFP cells (A-I). Cell viability after radiation (30 Gy) and various types and concentrations of anticancer agents in ACCS-M GFP, GFP ACCS-M shBr GFP, and ACCS-M shSOX2 GFP cells. Values are relative to non-irradiated ACCS-M GFP cells. Error bars indicate standard deviation. ${ }^{*} \mathrm{P}<0.001,{ }^{* * *} \mathrm{P}<0.05$

be effective for inhibiting cancer metastasis. It remains to be determined whether Brachyury knockdown in pre-existing cancer reduces the invasiveness of CSCs in the primary nest and increases their sensitivity to chemo- and radiotherapy.

Cellular invasiveness and migration were markedly higher in ACCS-M GFP than in ACCS GFP cells. Brachyury knockdown completely inhibited cellular invasiveness and migration, while SOX2 knockdown did not. Activation of cellular invasiveness is an important characteristic of EMT. Matrix metalloproteinases (MMPs) are upregulated in
EMT and induce cellular invasion of cancer cells (30-32). Moreover, EMT-related MMP-9 upregulation degrades cellsurface E-cadherin (33), an important phenotype of EMT. Thus, Brachyury knockdown inhibited not only tumorigenicity and metastasis, but also cancer cell invasion at the primary site. This finding suggests that Brachyury knockdown can inhibit cancer cell invasiveness of pre-existing cancers.

CSCs are chemoresistant. Similarly, ACCS-M GFP cells demonstrated resistance to all tested anticancer drugs except 
bleomycin. The mechanism underlying chemoresistance involves drug transporters. Genetic variations in efflux transporters of the ABC family, such as $A B C B 1$ (MDR1, P-glycoprotein), $A B C C 1$ (MRP1), $A B C C 2$ (MRP2), and $A B C G 2$ (BCRP), and uptake transporters of the SLC family, such as SLC19A1 (RFC1) and SLCO1B1 (SLC21A6), are implicated in resistance to chemotherapy $(34,35)$. We found that nearly all ABC family genes were expressed at higher levels in ACCS-M GFP than in ACCS GFP cells, and this difference in expression may underlie the chemoresistance of ACCS-M GFP cells. However, the degree of upregulation of these genes was not large $(\sim 5-15 \%)$, suggesting that another crucial factor underlies the chemoresistance of ACCS-M GFP.

Brachyury and SOX2 knockdown inhibited the expression of ABC family genes. The SOX2 gene enhances $A B C C 3$ and $A B C C 6$ expression through direct transcriptional regulation (36). Brachyury knockdown inhibited SOX2 expression. Therefore, Brachyury may indirectly inhibit ABC transporter genes thorough $S O X 2$ downregulation. By contrast, SLC family genes were upregulated in ACCS-M GFP cells, suggesting that drug uptake into cancer cells is induced in ACCS-M GFP cells. This finding contradicts the observed chemoresistance of ACCS-M GFP. However, only SLC19A1 (RFC1), the most relevant gene in chemoresistance $(37,38)$, was decreased in ACCS-M GFP cells, which indicates that drug uptake was inhibited. Furthermore, Brachyury knockdown recovered expression of SLC19A1 (RFC1) to its level in ACCS GFP cells, indicating that Brachyury knockdown induced drug uptake.

Another possible explanation for the drug resistance of CSCs is the characteristic proportion of CSCs at each stage of the cell cycle, because various anticancer drugs are cell cycle-dependent. CSCs have a significantly higher proportion of cells in the G2-phase of the cell cycle (39). Bleomycin, a glycopeptide antibiotic with a unique mechanism of antitumor activity, has G2-phase-specific cytotoxicity (40). Our results showed that only the cytotoxicity of bleomycin was unchanged by Brachyury knockdown in ACCS-M GFP cells. By contrast, ACCS-M GFP cells demonstrated resistance to the cell cyclespecific anticancer drugs 5-FU [S-phase (41)], etoposide [S/ G2-phase (42)], and taxanes [docetaxel and paclitaxel, G2/Mphase (43)], which was reduced by Brachyury knockdown. These results suggest that CSC resistance to cell cyclespecific anticancer agents is partially regulated by $\mathrm{G} 2$-phase elongation in CSCs and that Brachyury knockdown can break the cell cycle arrest in CSCs.

Cancer stem-like cells are relatively radioresistant owing to intrinsic and extrinsic factors, including quiescence, radiationresponse mechanisms (e.g., enhanced DNA repair, upregulated cell cycle-control mechanisms, and increased free-radical scavengers), and a microenvironment that enhances cell survival mechanisms (e.g., hypoxia and interaction with stromal elements) (44). Therefore, the same mechanisms of cell cycle regulation underlying chemoresistance of ACCS-M GFP or CSCs may contribute to radioresistance and the radiosensitizing effect of Brachyury knockdown. In radiation biology, cells in the late S-phase are especially resistant, and cells in the G2/Mphase are the most sensitive, to ionizing radiation (45). CSCs in the breast cancer cell line MDA-MB231 are shifted to the $\mathrm{S}$ - and G2-phases and are radioresistant. Cyclin D and E protein levels are consistent with this profile, suggesting the involvement of homologous recombination repair in the radioresistant phenotype (46). Therefore, cell cycle regulation in ACCS-M GFP cells may be a key factor underlying radioresistance. This mechanism is an important area of future investigation.

Clinically, CRT and multidrug chemotherapy reduce cancer cell viability by complementarily targeting cellular vulnerabilities. However, CSCs survive these treatments, because they do not target CSC cell cycle regulation. As shown in Fig. 8, Brachyury knockdown significantly enhanced the effect of CRT in vitro for all tested anticancer drugs to which cells were resistant as a single drug. These data support the conclusion from our previous study that Brachyury knockdown forcibly differentiates CSCs, causing them to lose their stemness. Furthermore, the effect of Brachyury knockdown was significantly stronger than that of $S O X 2$, a conventional stem cell regulatory gene. Multiple regulatory genes are believed to regulate cell stemness. However, we have shown that knockdown of a single gene, Brachyury, silenced multiple regulatory genes simultaneously. Hence, Brachyury knockdown may be an important therapeutic approach and should be further investigated for clinical use.

In conclusion, this study presents evidence that Brachyury knockdown reduces the invasiveness and chemo- and radioresistance of CSCs in vivo and suggests that Brachyury knockdown is a useful therapeutic tool for sensitizing CSCs to conventional chemoradiotherapy.

\section{Acknowledgements}

This study was supported by Grants-in-Aid from the Ministry of Education, Culture, Sports, Science, and Technology of Japan (KAKEN no. 23390465 to T. Sugiura and 25861958 to Y. Kobayashi). We would like to thank Dr Manabu Kawata (Institute of Microbial Chemistry, Numazu Microbial Chemistry Research Foundation) for providing standard anticancer drug kits.

\section{References}

1. Rapidis AD, Givalos N, Gakiopoulou H, et al: Adenoid cystic carcinoma of the head and neck. Clinicopathological analysis of 23 patients and review of the literature. Oral Oncol 41: 328-335, 2005.

2. Ampil FL and Misra RP: Factors influencing survival of patients with adenoid cystic carcinoma of the salivary glands. J Oral Maxillofac Surg 45: 1005-1010, 1987.

3. Ishii K, Shimoda M, Sugiura T, et al: Involvement of epithelialmesenchymal transition in adenoid cystic carcinoma metastasis. Int J Oncol 38: 921-931, 2011

4. Shimoda M, Sugiura T, Imajyo I, et al: The T-box transcription factor Brachyury regulates epithelial-mesenchymal transitions in association with cancer stem-like cells in adnoid cystic carcinoma cells. BMC Cancer 12: 377, 2012.

5. Grunert S, Jechlinger $M$ and Beug H: Diverse cellular and molecular mechanisms contribute to epithelial plasticity and metastasis. Nat Rev Mol Cell Biol 4: 657-665, 2003.

6. Cano A, Perez-Moreno MA, Rodrigo I, et al: The transcription factor snail controls epithelial-mesenchymal transitions by repressing E-cadherin expression. Nat Cell Biol 2: 76-83, 2000.

7. Yang MH, Hsu DS, Wang HW, et al: Bmil is essential in Twist1induced epithelial-mesenchymal transition. Nat Cell Biol 12: 982-992, 2010

8. Kispert A, Herrmann BG, Leptin M and Reuter R: Homologs of the mouse Brachyury gene are involved in the specification of posterior terminal structures in Drosophila, Tribolium, and Locusta. Genes Dev 8: 2137-2150, 1994. 
9. Behr R, Heneweer C, Viebahn C, Denker HW and Thie M: Epithelial-mesenchymal transition in colonies of rhesus monkey embryonic stem cells: a model for processes involved in gastrulation. Stem Cells 23: 805-816, 2005.

10. Vidricaire G, Jardine K and McBurney MW: Expression of the Brachyury gene during mesoderm development in differentiating embryonal carcinoma cell cultures. Development 120: 115-122, 1994.

11. Fernando RI, Litzinger M, Trono P, Hamilton DH, Schlom J and Palena C: The T-box transcription factor Brachyury promotes epithelial-mesenchymal transition in human tumor cells. J Clin Invest 120: 533-544, 2010.

12. Reya T, Morrison SJ, Clarke MF and Weissman IL: Stem cells, cancer, and cancer stem cells. Nature 414: 105-111, 2001.

13. Thiery JP: Epithelial-mesenchymal transitions in development and pathologies. Curr Opin Cell Biol 15: 740-746, 2003.

14. Mani SA, Guo W, Liao MJ, et al: The epithelial-mesenchymal transition generates cells with properties of stem cells. Cell 133: 704-715, 2008

15. Blick T, Hugo H, Widodo E, et al: Epithelial mesenchymal transition traits in human breast cancer cell lines parallel the CD44(hi/)CD24 (lo/-) stem cell phenotype in human breast cancer. J Mammary Gland Biol Neoplasia 15: 235-252, 2010.

16. Huang HP, Chen PH, Yu CY, et al: Epithelial cell adhesion molecule (EpCAM) complex proteins promote transcription factor-mediated pluripotency reprogramming. J Biol Chem 286: 33520-33532, 2011.

17. Utikal J, Maherali N, Kulalert W and Hochedlinger K: Sox 2 is dispensable for the reprogramming of melanocytes and melanoma cells into induced pluripotent stem cells. J Cell Sci 122: 3502-3510, 2009

18. Ahmed N, Abubaker K, Findlay J and Quinn M: Epithelial mesenchymal transition and cancer stem cell-like phenotypes facilitate chemoresistance in recurrent ovarian cancer. Curr Cancer Drug Targets 10: 268-278, 2010.

19. Alison MR, Lim SM and Nicholson LJ: Cancer stem cells: problems for therapy? J Pathol 223: 147-161, 2010.

20. Wang Z, Li Y, Ahmad A, Azmi AS, Kong D, Banerjee S and Sarkar FH: Targeting miRNAs involved in cancer stem cell and EMT regulation: an emerging concept in overcoming drug resistance. Drug Resist Updat 13: 109-118, 2010.

21. Raimondi C, Gianni W, Cortesi E and Gazzaniga P: Cancer stem cells and epithelial-mesenchymal transition: revisiting minimal residual disease. Curr Cancer Drug Targets 10: 496-508, 2010

22. Herrmann BG, Labeit S, Poustka A, King TR and Lehrach $H$ Cloning of the $\mathrm{T}$ gene required in mesoderm formation in the mouse. Nature 343: 617-622, 1990.

23. Edwards YH, Putt W, Lekoape KM, Stott D, Fox M, Hopkinson DA and Sowden J: The human homolog T of the mouse T(Brachyury) gene; gene structure, cDNA sequence, and assignment to chromosome 6q27. Genome Res 6: 226-233, 1996.

24. Sarkar D, Shields B, Davies ML, Muller J and Wakeman JA: BRACHYURY confers cancer stem cell characteristics on colorectal cancer cells. Int J Cancer 130: 328-337, 2012

25. Abe M, Sugiura T, Takahashi M, Ishii K, Shimoda M and Shirasuna K: A novel function of CD82/KAI-1 on E-cadherinmediated homophilic cellular adhesion of cancer cells. Cancer Lett 266: 163-170, 2008.

26. Brown AM: A step-by-step guide to non-linear regression analysis of experimental data using a Microsoft Excel spreadsheet. Comput Methods Programs Biomed 65: 191-200, 2001.

27. Hsu HS, Huang PI, Chang YL, et al: Cucurbitacin I inhibits tumorigenic ability and enhances radiochemosensitivity in nonsmall cell lung cancer-derived CD133-positive cells. Cancer 117: 2970-2985, 2011

28. Cheung ST, Cheung PF, Cheng CK, Wong NC and Fan ST: Granulin-epithelin precursor and ATP-dependent binding cassette (ABC)B5 regulate liver cancer cell chemoresistance. Gastroenterology 140: 344-355, 2011.
29. Chuthapisith S, Eremin J, El-Sheemey M and Eremin O: Breast cancer chemoresistance: emerging importance of cancer stem cells. Surg Oncol 19: 27-32, 2011.

30. Hayashi Y, Osanai M and Lee GH: Fascin-1 expression correlates with repression of E-cadherin expression in hepatocellular carcinoma cells and augments their invasiveness in combination with matrix metalloproteinases. Cancer Sci 102: 1228-1235, 2011.

31. Lin CY, Tsai PH, Kandaswami CC, Lee PP, Huang CJ, Hwang JJ and Lee MT: Matrix metalloproteinase-9 cooperates with transcription factor Snail to induce epithelial-mesenchymal transition. Cancer Sci 102: 815-827, 2011.

32. Zhang K, Chen D, Jiao X, et al: Slug enhances invasion ability of pancreatic cancer cells through upregulation of matrix metalloproteinase-9 and actin cytoskeleton remodeling. Lab Invest 91: 426-438, 2011.

33. Zuo JH, Zhu W, Li MY, et al: Activation of EGFR promotes squamous carcinoma SCC10A cell migration and invasion via inducing EMT-like phenotype change and MMP-9-mediated degradation of E-cadherin. J Cell Biochem 112: 2508-2517, 2011.

34. Huang Y and Sadee W: Membrane transporters and channels in chemoresistance and -sensitivity of tumor cells. Cancer Lett 239: 168-182, 2006.

35. Huang Y: Pharmacogenetics/genomics of membrane transporters in cancer chemotherapy. Cancer Metastasis Rev 26: 183-201, 2007.

36. Jeon HM, Sohn YW, Oh SY, Kim SH, Beck S, Kim S and Kim H: ID4 imparts chemoresistance and cancer stemness to glioma cells by derepressing miR-9*-mediated suppression of SOX 2 . Cancer Res 71: 3410-3421, 2011.

37. Yang R, Li WW, Hoang BH, et al: Quantitative correlation between promoter methylation and messenger RNA levels of the reduced folate carrier. BMC Cancer 8: 124, 2008.

38. Matherly LH, Hou Z and Deng Y: Human reduced folate carrier: translation of basic biology to cancer etiology and therapy. Cancer Metastasis Rev 26: 111-128, 2007.

39. Harper LJ, Costea DE, Gammon L, Fazil B, Biddle A and Mackenzie IC: Normal and malignant epithelial cells with stem-like properties have an extended G2 cell cycle phase that is associated with apoptotic resistance. BMC Cancer 10:166, 2010.

40. Dorr RT: Bleomycin pharmacology: mechanism of action and resistance, and clinical pharmacokinetics. Semin Oncol 19: 3-8, 1992.

41. Song B, Wang Y, Xi Y, et al: Mechanism of chemoresistance mediated by miR-140 in human osteosarcoma and colon cancer cells. Oncogene 28: 4065-4074, 2009.

42. Lin CK, Nguyen TT, Morgan TL, et al: Apoptosis may be either suppressed or enhanced with strategic combinations of antineoplastic drugs or anti-IgM. Exp Cell Res 244: 1-13, 1998.

43. O'Leary J, Volm M, Wasserheit C and Muggia F: Taxanes in adjuvant and neoadjuvant therapies for breast cancer. Oncology (Williston Park) 12: 23-27, 1998.

44. Hittelman WN, Liao Y, Wang L and Milas L: Are cancer stem cells radioresistant? Future Oncol 6: 1563-1576, 2010

45. Pajonk F, Vlashi E and McBride WH: Radiation resistance of cancer stem cells: the 4 R's of radiobiology revisited. Stem Cells 28: 639-648, 2010

46. Al-Assar O, Mantoni T, Lunardi S, Kingham G, Helleday T and Brunner TB: Breast cancer stem-like cells show dominant homologous recombination due to a larger S-G2 fraction. Cancer Biol Ther 11: 1028-1035, 2011. 